Fast frequency response computation for Rayleigh damping

\author{
Karl Meerbergen
}

Report TW 480, November 2006

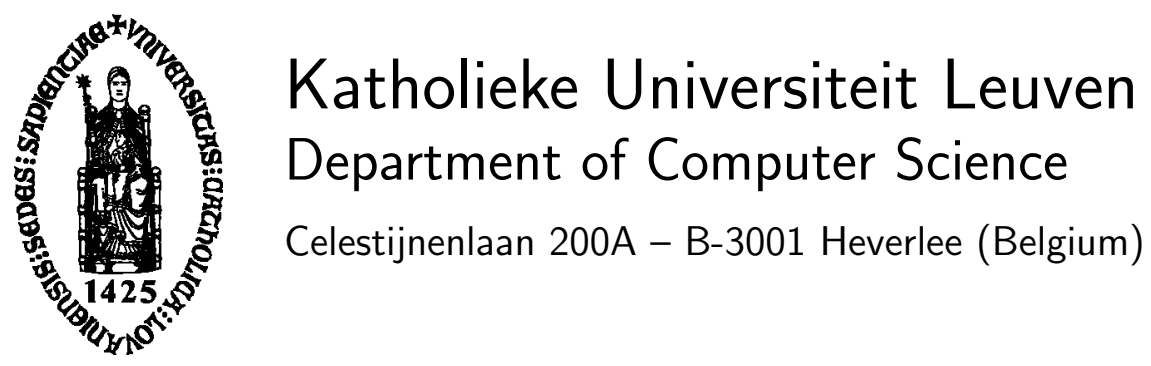




\title{
Fast frequency response computation for Rayleigh damping
}

\author{
Karl Meerbergen \\ Report TW 480, November 2006
}

Department of Computer Science, K.U.Leuven

\begin{abstract}
We present a model reduction Lanczos method for the computation of frequency response functions of Rayleigh damping problems. The connection with modal extraction and modal superposition is made. The usual model reduction methods require complex arithmetic. With the proposed method, complex arithmetic is only used for the reduced problem. A numerical example from structural damping is given.
\end{abstract}

Keywords : frequency response function, Lanczos method, modal superposition, iterative methods, model reduction.

AMS(MOS) Classification : 65F10, 74S05 


\title{
Fast frequency response computation for Rayleigh damping
}

\author{
Karl Meerbergen*
}

November 30, 2006

\begin{abstract}
We present a model reduction Lanczos method for the computation of frequency response functions of Rayleigh damping problems. The connection with modal extraction and modal superposition is made. The usual model reduction methods require complex arithmetic. With the proposed method, complex arithmetic is only used for the reduced problem. A numerical example from structural damping is given.
\end{abstract}

Keywords: frequency response function, Lanczos method, modal superposition, iterative methods, model reduction.

\section{Introduction}

Consider the system

$$
\left(K-\omega^{2} M+D(\omega)\right) x=f
$$

for $\omega=\omega_{1}, \ldots, \omega_{m}$. Problems of this form arise in the computation of the frequency response function of vibration problems, discretized by finite elements. The matrices $M$ and $K$ in $\mathbf{R}^{n \times n}$ are the mass and stiffness matrices respectively, and are symmetric and positive definite [7] [6]. Often, $K$ is positive semi-definite. The matrix $D$ is a damping matrix and is also symmetric but complex. In this paper, we study the case of Rayleigh damping

$$
D(\omega)=\gamma(\omega) K+\delta(\omega) M
$$

This a special case of modal damping, i.e. there are $U \in \mathbf{R}^{n \times n}$ so that

$$
U^{*} K U=\Lambda^{2} \quad, \quad U^{*} M U=I \quad, \quad U^{*} D U=\Delta
$$

where $\Lambda=\operatorname{diag}\left(\lambda_{1}, \ldots, \lambda_{n}\right)$ and $\Delta=\operatorname{diag}\left(\delta_{1}, \ldots, \delta_{n}\right)$ are diagonal matrices. The columns of $U$ are eigenvectors. Rayleigh damping is a physical model that is actively studied over a long period [13] [14] [17]. The most popular method for solving models with Rayleigh damping is modal superposition, i.e. the matrices $K, M$ and $D$ are diagonalized by right and left multiplication with well selected columns of $U$. This reduces (1) into a diagonal linear system. Sometimes complex systems are approximated by Rayleigh or proportional damping models [2]. The major disadvantage of this approach is that a relatively large number of eigenvalues and eigenvectors of $(K, M)$ may have to be computed. This is usually accomplished by the block Lanczos method [15, 16, 19].

In this paper, we propose the Lanczos method that is very close to modal superposition but can be more efficient in terms of memory. The method is significantly easier to implement than modal extraction. In general less vectors are required as well, since the method focuses on computing only the modes that have important contributions to the solution. This type of method has been successfully applied in electronic circuit design [10] [3] and for undamped structures [20], and exterior acoustic problems [18]. See [8] for an overview of model reduction methods.

In the literature, methods are proposed when $\gamma$ and $\delta$ are a linear function of $\omega$. We then have a problem of the form

$$
\left(A_{0}+\omega A_{1}+\omega^{2} A_{2}\right) x=f
$$

*Katholieke Universiteit Leuven, Department of Computer Science, Celestijnenlaan 200A, 3001 Heverlee, Belgium. Karl.Meerbergen@cs.kuleuven. be 


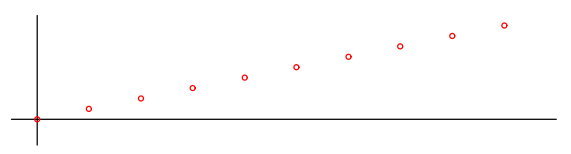

a. Structural damping

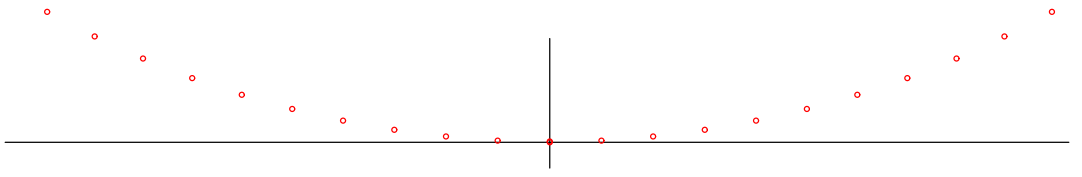

b. Fluid damping

Figure 1: Spectrum for Rayleigh damping

where $A_{0}, A_{1}$ or $A_{2}$ are complex symmetric. Krylov methods for polynomials of degree two in $\omega$ are well suited, e.g. [5] [4]. When $\gamma$ and $\delta$ are higher degree polynomials in $\omega$, we can use [11]. These methods can be expensive for large degree polynomials. Moreover, the construction of the Krylov subspaces requires complex arithmetic. In this paper, we present a method based on the Lanczos method that builds a Krylov space in real arithmetic, which leads to significant reductions in computation time and memory.

Since $K$ often is singular, $K$ is shifted into $K_{\sigma}=K-\sigma^{2} M$ with $K-\sigma^{2} M$ non-singular. The Lanczos method relies on the existence of such a $\sigma$. (Note that when $M$ and $K$ have a common nullspace, this condition is too strong. In this case, it is not obvious how to solve linear systems with $K-\sigma^{2} M$ and therefore we do no longer consider this case. See [9] for more details on the spectral structure.)

In this paper, we extend the Lanczos method for the solution of undamped systems to systems with Rayleigh damping. The Lanczos method has connections with the conjugate gradient method [20]. With the presence of damping, these properties slightly change. The connection with Padé approximation [10, 3] is less helpful in the context of this manuscript.

We use $x^{*} y$ to denote the Euclidean inner product of $x$ and $y$ and by $\|x\|$ or $\|x\|_{2}$, we denote the induced norm. We also use the inner product $x^{*} M y$ and the induced norm $\|x\|_{M}=\sqrt{x^{*} M x}$.

Here is the plan of the paper. In $\S 2$, we define proportional and Rayleigh damping and show properties. In $\S 3$, we introduce the Lanczos method and its use for the solution of problems with Rayleigh damping. Section 4 shows the connection with iterative methods and the vector Padé approximation. The convergence is similar to the undamped case, but the damping may turn the residual of (1) quicker to zero. The theory is illustrated in $\S 5$ for a vibrating windscreen, which is an example of structural damping. We finally summarize the conclusion in $\S 6$.

\section{Proportional damping}

In this section, we describe a number of cases of proportional damping.

Structural damping takes the form

$$
D \equiv i \gamma K
$$

The spectrum of

$$
(1+i \gamma) K u=\lambda^{2} M u
$$

lies on a line in the complex plane as shown by Figure 1.a. We obtain the equation

$$
\left\{(1+i \gamma) K-\omega^{2} M\right\} x=f .
$$

Fluid damping takes the form

$$
D(\omega)=i \omega(\gamma K+\delta M) .
$$

The corresponding eigenvalue problem is quadratic, where each eigenvector has two distinct eigenvalues. The spectrum of the quadratic eigenvalue problem

$$
K u+i \lambda(\gamma K+\delta M) u-\lambda^{2} M u=0
$$

lies on a curve in the complex plane as shown by Figure 1.b. We then obtain

$$
\left\{K+i \omega(\gamma K+\delta M)-\omega^{2} M\right\} x=f .
$$


It is clear that both forms of Rayleigh damping are modal. A more general form of Rayleigh damping is called proportional damping where

$$
D(\omega)=\sum_{j=0}^{q} \beta_{j} M\left(M^{-1} K\right)^{j} .
$$

In this paper, we use a different form of proportional damping which is closer to the numerical method that is proposed :

$$
D(\omega)=\sum_{j=0}^{p} \alpha_{j} K_{\sigma}\left(K_{\sigma}^{-1} M\right)^{j} .
$$

The following theorem shows the connection between modal damping and proportional damping.

Theorem 1 Suppose that there is an $n \times n$ matrix $U$ so that $U^{*} M U=I, U^{*} K U=\Lambda^{2}$ diagonal and $U^{*} D(\omega) U=$ $\Delta(\omega)$ diagonal. Assume that if $\lambda_{j}=\lambda_{i}$, then $\delta_{i}=\delta_{j}$. Then there are $p \leq n$ and $\alpha_{j}$ for $j=0, \ldots, p$ so that (9) holds.

Proof. First note that $U^{*} M K_{\sigma}^{-1} M U=\Theta$ with

$$
\Theta=\left(\Lambda^{2}-\sigma^{2} I\right)^{-1}=\operatorname{diag}\left(\theta_{1}, \ldots, \theta_{n}\right) .
$$

Let $p$ be the number of distinct eigenvalues. Consider the linear system in $\alpha_{0}, \ldots, \alpha_{p}$ :

$$
\delta_{i}=\sum_{j=0}^{p} \alpha_{j} \theta_{i}^{j-1} \quad i=1, \ldots, n .
$$

This is a Vandermonde linear system in $\alpha_{0}, \ldots, \alpha_{p}$. If $p \lambda_{j}$ 's are distinct, this is a non-singular system. If $\delta_{i}=\delta_{j}$ when $\lambda_{j}=\lambda_{i}$, the right hand side is consistent and so we have a solution. From (10), we derive

$$
\Delta=\sum_{j=0}^{p} \alpha_{j} \Theta^{j-1}
$$

Next, we note that

$$
D=U^{-*} \Delta U^{-1}
$$

and

$$
\sum_{j=0}^{p} \alpha_{j} U^{-*} \Theta^{j-1} U^{-1}=\sum_{j=0}^{p} K_{\sigma}\left(K_{\sigma}^{-1} M\right)^{j} .
$$

The proof follows from combining (11), (12) and (13).

As we shall see later, there is an advantage for having small $p$. For arbitrary modal damping, $p$ can be very large, so that the proposed method is not necessarily efficient. Recall that for structural Rayleigh damping, $p=0$ and for fluid Rayleigh damping, $p=1$. We mainly concentrate on these two cases.

We just give a small example to illustrate Theorem 1. Let

$$
K=\left[\begin{array}{ccc}
2 & & \\
& 1 & \\
& & 1
\end{array}\right] \quad M=I
$$

and let $\delta_{1}=i 0.1$ and $\delta_{2,3}=0.2 i$. Since $U=I$, we know that $D=\Delta$, and since we have two distinct eigenfrequencies, $p 12$. We are now seeking $\alpha_{0}, \alpha_{1}$ so that $\delta_{i}=\alpha_{0} \lambda_{i}^{2}+\alpha_{1}$. For $i=1,2,3$, we find $\alpha_{0}=-0.1 i$ and $\alpha_{1}=0.3 i$. 


\section{The Lanczos method}

In this section, we introduce the Lanczos method for the matrix pair $(K, M)$, see [15] [16] [20].

Here is an outline of the method.

1. Compute the initial vector $w_{1}=K_{\sigma}^{-1} f$ and normalize into $v_{1}=w_{1} /\left\|v_{1}\right\|_{M}$. Let $v_{0}=0$ and $\beta_{0}=0$.

2. For $j=1, \ldots, k$

2.1. Compute Krylov vector $w_{j+1}=K_{\sigma}^{-1} M v_{j}$.

2.2. compute $\gamma_{j}=v_{j}^{*} M w_{j+1}$

2.3 Update: $w_{j+1} \leftarrow w_{j+1}-\beta_{j-1} v_{j-1}-\gamma_{j} v_{j}$

2.4 Normalization: $\beta_{j}=\left\|w_{j+1}\right\|_{M}$ and $v_{j+1}=w_{j+1} / \beta_{j}$.

The Lanczos method produces a set of vectors $V_{k+1}=\left[v_{1}, \ldots, v_{k+1}\right]$ and a $k \times k$ symmetric tridiagonal matrix $T_{k}$ whose diagonal entries are $\gamma_{1}, \ldots, \gamma_{k}$ and whose off diagonal elements are $\beta_{1}, \ldots, \beta_{k-1}$. They satisfy the relation

$$
K_{\sigma}^{-1} M V_{k}-V_{k} T_{k}=\beta_{k} v_{k+1} e_{k}^{*} .
$$

Let $\theta$ be an eigenvalue of $T_{k}$. Typically the large eigenvalues $\theta$ are good approximations to eigenvalues of $K_{\sigma}^{-1} M$. This property is used to compute eigenvalues of the undamped problem. It is the basis of the Lanczos method for modal extraction [15]. If the right-hand side in (14) is zero, the eigenvalues of $T_{k}$ are exact eigenvalues of $K_{\sigma}^{-1} M$.

The vectors $v_{1}, \ldots, v_{k+1}$ span the Krylov subspace $\operatorname{span}\left\{v_{1}, K_{\sigma}^{-1} M v_{1}, \ldots\left(K_{\sigma}^{-1} M\right)^{k} v_{1}\right\}$. This property makes the Lanczos method a Krylov method. Krylov subspaces play an important role in iterative methods in linear algebra.

Steps 2.2-2.4 ensure $M$ orthogonality of the columns of $V_{k}$ in exact arithmetic, i.e. $V_{k}^{*} M V_{k}=I$. Due to rounding errors, orthogonality is lost and, therefore, it may be advantageous to reorthogonalize the Lanczos vectors in order to ensure orthogonality [15], although this is not required for the solution of (1) [20].

The solution of (1) is now approximated by projection on the subspace spanned by the columns of $V_{k}$ :

$$
V_{k}^{*} M K_{\sigma}^{-1}\left(K_{\sigma}-\left(\omega^{2}-\sigma^{2}\right) M+D(\omega)\right) V_{k} z=\left\|w_{1}\right\|_{M} e_{1}
$$

and compute $x=V_{k} z$. This is a $k \times k$ linear system whose solution is very cheap if $k$ is significantly smaller than $n$. The number $k$ is much smaller then $n$, typically smaller than one hundred. Since there is, a priori, no relation between $K_{\sigma}^{-1} D$ and $K_{\sigma}^{-1} M$, there is no reason why (15) produces accurate results for (1). We shall see, however, that it does make sense for Rayleigh damping.

The important points here are

- that the Lanczos method (the more expensive part) is carried out in real arithmetic and the $k \times k$ system (15) in complex arithmetic,

- and that $D(\omega)$ may depend on $\omega$.

\section{Analysis}

We first analyze the situation for Rayleigh damping and then show some results for a more general situation.

Another way to solving (1) is to apply an iterative method for solving

$$
K_{\sigma}^{-1}\left(K-\omega^{2} M+D(\omega)\right) x=K_{\sigma}^{-1} f
$$

for any value of $\omega$.

In [12] [21] [20], it was shown that the Krylov subspace is independent of $\omega \neq \sigma$ when $D \equiv 0$ and therefore it is sufficient to compute it only once, using the Lanczos method. For Rayleigh damping we have a similar situation as we now show.

\subsection{Rayleigh damping}

From $(2)$, we have $K_{\sigma}^{-1} D(\omega)=\gamma(\omega) I+\left(\delta(\omega)+\gamma(\omega) \sigma^{2}\right) K_{\sigma}^{-1} M$, so

$$
A=K_{\sigma}^{-1}\left(K-\omega^{2} M+D(\omega)\right)=(1+\gamma) I+\left(\delta+\gamma \sigma^{2}-\omega^{2}+\sigma^{2}\right) K_{\sigma}^{-1} M .
$$


The Krylov subspaces for $K_{\sigma}^{-1} M$ and $A$ are the same if $\delta+\gamma \sigma^{2}-\omega^{2}+\sigma^{2} \neq 0$. From (14), we derive that

$$
A V_{k}-V_{k}\left((1+\gamma) I+\left(\delta+\gamma \sigma^{2}-\omega^{2}+\sigma^{2}\right) T_{k}\right)=\left(\delta+\gamma \sigma^{2}-\omega^{2}+\sigma^{2}\right) \beta_{k} v_{k+1} e_{k}^{*} .
$$

This is the recurrence relation that we would obtain if we would apply the Lanczos method directly to $A$. This shows that we do not have to construct the Krylov subspace for any new value of $\omega$. This property is valid in exact arithmetic. A rounding error analysis was performed in [20] in the case of undamped problems. A more accurate solution could perhaps be obtained when applying the Lanczos method to $A$ for all values of $\omega$ of interest. Of course, such an approach would be much more expensive.

\subsection{Connection with iterative methods}

Let $K u=\lambda^{2} M u$ with $\|u\|_{M}=1$ denote the eigenvalue problem. Denote the eigenpairs by $\left(\lambda_{j}, u_{j}\right)$. The eigenpairs of $A$ have the form $\left(\mu_{j}, u_{j}\right)$ with

$$
\begin{aligned}
\mu_{j} & =\frac{\lambda_{j}^{2}-\omega^{2}+\delta+\gamma \lambda_{j}^{2}}{\lambda_{j}^{2}-\sigma^{2}} \\
& =(1+\gamma)+\frac{\delta+\gamma \sigma^{2}}{\lambda_{j}^{2}-\sigma^{2}}+\frac{\sigma^{2}-\omega^{2}}{\lambda_{j}^{2}-\sigma^{2}}
\end{aligned}
$$

Most $\lambda_{j}$ lie far away from $\sigma$. we conclude that when $\omega \simeq \sigma$, the spectrum of $A$ is clustered around $1+\gamma$ with possibly a few eigenvalues near zero depending on $\omega$. This is favourable for an iterative Krylov solver to converge fast. When $\omega^{2}-\sigma^{2}$ becomes large, the clustering decreases, since the last term in (18) is no longer small for most eigenvalues. So convergence is slower.

\subsection{Characterization of the convergence}

We define the Ritz pair $(\tilde{\mu}, \tilde{u})$ of a matrix $B$ as $\tilde{u}=V_{k} z$ with $V_{k}^{*} M A V_{k} z=\tilde{\mu} z$ and $\|z\|_{2}=1$. The Ritz pairs of $K_{\sigma}^{-1} M,\left(\tilde{\lambda}_{j}^{2}-\sigma^{2}\right)^{-1}$ are good approximations to the large $\left(\lambda_{j}^{2}-\sigma^{2}\right)^{-1}$, i.e. the $\lambda$ 's near the shift point $\sigma$ are well approximated.

Let $\left(\tilde{\mu}_{j}, \tilde{u}_{j}\right)$ for $j=1, \ldots, k$ be the Ritz pairs of $A$. The Ritz vectors $\tilde{u}_{j}$ are the same for $A$ and $K_{\sigma}^{-1} M$ and the Ritz values are

$$
\tilde{\mu}_{j}=\frac{\tilde{\lambda}_{j}^{2}-\omega^{2}+\delta+\gamma \tilde{\lambda}_{j}^{2}}{\tilde{\lambda}_{j}^{2}-\sigma^{2}} .
$$

Following (15), the solution can be written in the form:

$$
\tilde{x}(\omega)=\sum_{j=1}^{k} \tilde{u}_{j} \frac{\tilde{u}_{j}^{*} M K_{\sigma}^{-1} f}{\tilde{\mu}_{j}(\omega)}
$$

This looks very similar to modal superposition.

In [20], we have shown for the undamped problem the following results, which also hold for Rayleigh damping :

Proposition 1 Let $r$ be the residual

$$
r=K_{\sigma}^{-1}(f-A \tilde{x})
$$

then

$$
\|r\|_{2} \leq \sum_{j=1}^{k} \beta_{k}\left|\tilde{u}_{j}^{*} M f\right| \frac{\rho_{j}}{\left|\tilde{\mu}_{j}\right|}
$$

with $\rho_{j}=\left\|A \tilde{u}_{j}-\tilde{\mu}_{j} \tilde{u}_{j}\right\|_{M}$. 
Proof. The proof follows the logic from the proof of Lemma 4.1 in [20].

We note that the residual is small when either $\rho_{j} /\left|\tilde{\mu}_{j}\right|$ or $\left|\tilde{u}_{j}^{*} M f\right|$ are small. Small $\mu_{j}$ need to be computed more accurately than large ones. Small $\mu_{j}$ correspond to peaks in the frequency response function. The positions of these peaks need to be computed accurately.

We know that the Lanczos method computes the $\lambda_{j}$ 's near $\sigma$ accurately. Only the small $\mu_{j}$ 's need to be computed accurately. When damping is low, these correspond to the $\lambda_{j}$ 's near $\omega$. It indicates that a larger Krylov subspace (larger $k$ ) is needed for $\omega$ away from $\sigma$, because computing $\lambda_{j}$ 's near $\omega$ away from $\sigma$ requires larger subspaces. When modal damping is large (large $\delta$ ), the accuracy of the eigenvalue is less important.

This also shows a connection with modal superposition. The spectral density also plays a role in the number of modes (i.e. vectors) to be used. When the density of eigenvalues is high, a large number of eigenvalues must be computed, and so a large Krylov subspace be built.

Note that if $\left|\delta+\gamma \sigma^{2}-\omega^{2}+\sigma^{2}\right| /|1+\gamma|$ is small, then $A \approx I$. The Lanczos method then produces accurate results, even for a small number of vectors. This situation only happens when $\delta$ and $\gamma$ are small, and $\sigma$ is close to $\omega$.

\subsection{Connection to vector Padé}

Consider the situation of Rayleigh damping with $\gamma$ independent of $\omega$. Define

$$
\alpha=\frac{\delta+\gamma \sigma^{2}+\sigma^{2}-\omega^{2}}{1+\gamma}
$$

then (1) can be written as

$$
(I+\alpha B) x=b
$$

with $B=K_{\sigma}^{-1} M$ and $b=\frac{1}{1+\gamma} f$. The Lanczos method for this problem is well understood. The connection with the vector-Padé approximation is known, see [10][3][20].

Theorem 2 Develop

$$
\begin{aligned}
& x=x_{0}+\alpha x_{1}+\cdots+\alpha^{j} x_{j}+\cdots \\
& \tilde{x}=\tilde{x}_{0}+\alpha \tilde{x}_{1}+\cdots+\alpha^{j} \tilde{x}_{j}+\cdots
\end{aligned}
$$

then

$$
x_{j}=\tilde{x}_{j} \quad \text { for } \quad j=0, \ldots, k-1 .
$$

Proof. See [10][3][20].

Especially when $\alpha$ is close to zero, the Lanczos approximation is likely to be very accurate. For the undamped case, $\alpha \simeq 0$ when $\omega \simeq \sigma$. Unfortunately, $|\alpha|$ can be large for Rayleigh damping: it depends on the values of $\delta$ and $\gamma$. This makes the connection with the Padé approximation less useful as for the undamped case.

\subsection{Proportional damping}

The situation is more complicated, since $A$ now takes the form

$$
A=\alpha_{0} I+\alpha_{1} K_{\sigma}^{-1} M+\cdots+\alpha_{p}\left(K_{\sigma}^{-1} M\right)^{p} .
$$

Note that $\alpha_{0} I+\alpha_{1} K_{\sigma}^{-1} M$ contains the terms $K_{\sigma}^{-1}\left(K-\omega^{2} M\right)$. Let $B=K_{\sigma}^{-1} M$. Define $\underline{T}_{k}=\left(\begin{array}{c}T_{k} \\ \beta_{k} e_{k}^{*}\end{array}\right)$. Then for $p \geq 2$, we have

$$
\begin{aligned}
B V_{k} & =V_{k+1} \underline{T}_{k} \\
B^{2} V_{k-1} & =V_{k+1} \underline{T}_{k} \underline{T}_{k-1} \\
& \cdots \\
B^{p-1} V_{k-p+1} & =V_{k+1} \underline{T}_{k} \cdots \underline{T}_{k-p+1}
\end{aligned}
$$




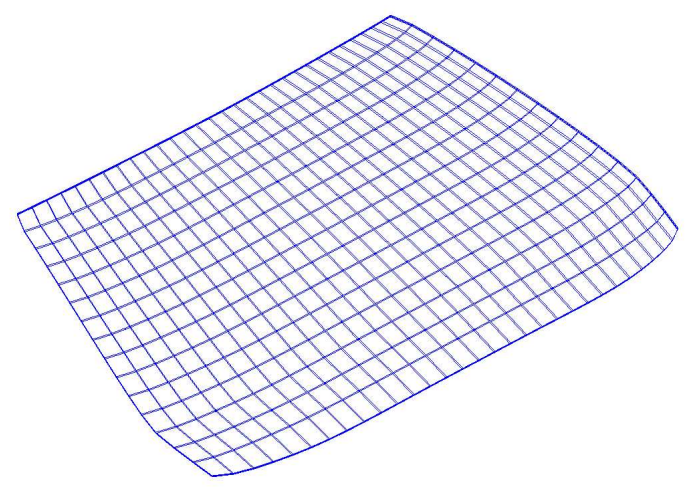

Figure 2: Mesh of the car windscreen

from which we can cheaply compute $V_{k}^{*} M B V_{k}, \ldots, V_{k-p+1}^{*} M B^{p-1} V_{k-p+1}$. Hence $V_{k-p+1}^{*} M A V_{k-p+1}$ is computed from (20). So, we must perform $p-1$ additional Lanczos iterations for computing $V_{k}^{*} M A V_{k}$ using the Lanczos method.

We do not explore this idea any further since it is not the objective of the paper.

\section{Numerical example}

The test problem illustrates the method on a structural model of a car windscreen. This is a 3D problem discretized with 7564 nodes and 5400 linear hexahedral elements HEX08 (3 layers of $60 \times 30$ elements) [1]. The mesh is shown in Figure 2. The material is glass with the following properties: the Young modulus is $710^{10} \mathrm{~N} / \mathrm{m}^{2}$, the density is $2490 \mathrm{~kg} / \mathrm{m}^{3}$, and the Poisson ratio is 0.23 . The natural damping is $10 \%$. This corresponds to $\gamma=0.1$. The structural boundaries are free (free-free boundary conditions). The plate is subjected to a point force applied on a corner node.

The discretized problem has dimension $n=22,692$. The goal is to estimate $x(\omega)$ with $\omega \in[0.5,200]$. In order to generate the plots the frequency range was discretized as $\left\{\omega_{1}, \ldots, \omega_{m}\right\}=\{0.5 j, j=1, \ldots, m\}$ with $m=400$.

We ran the Lanczos method with $\sigma=200$ and $k=20$. This run costed 14 seconds of which 9 were spent in running the Lanczos process. Figure 3 shows the exact solution norm $\|x\|_{2}$ and the error norm $\|x-\tilde{x}\|_{2}$ for the Lanczos method.

When we use $\sigma=0.5$ and $k=40$, there is no visual difference between the frequency response function from the direct computation and the Lanczos method. Figure 4 shows the exact solution norm $\|x\|_{2}$ and the error norm $\|x-\tilde{x}\|_{2}$. The computation of the exact solution required complex arithmetic and costed 2653 seconds computation time. The Lanczos method required only 26 seconds, of which 20 seconds were required for the construction of the Lanczos process.

\section{Conclusions}

We have presented a model reduction method that exploits the spectral structure of Rayleigh damping. This allows us to use the symmetric Lanczos method with real arithmetic for problems with complex valued damping matrices that may be strongly frequency dependent.

\section{References}

[1] Oberwolfach model reduction benchmark collection, $2004 . \quad$ http://www.imtek.unifreiburg.de/simulation/benchmark/. 


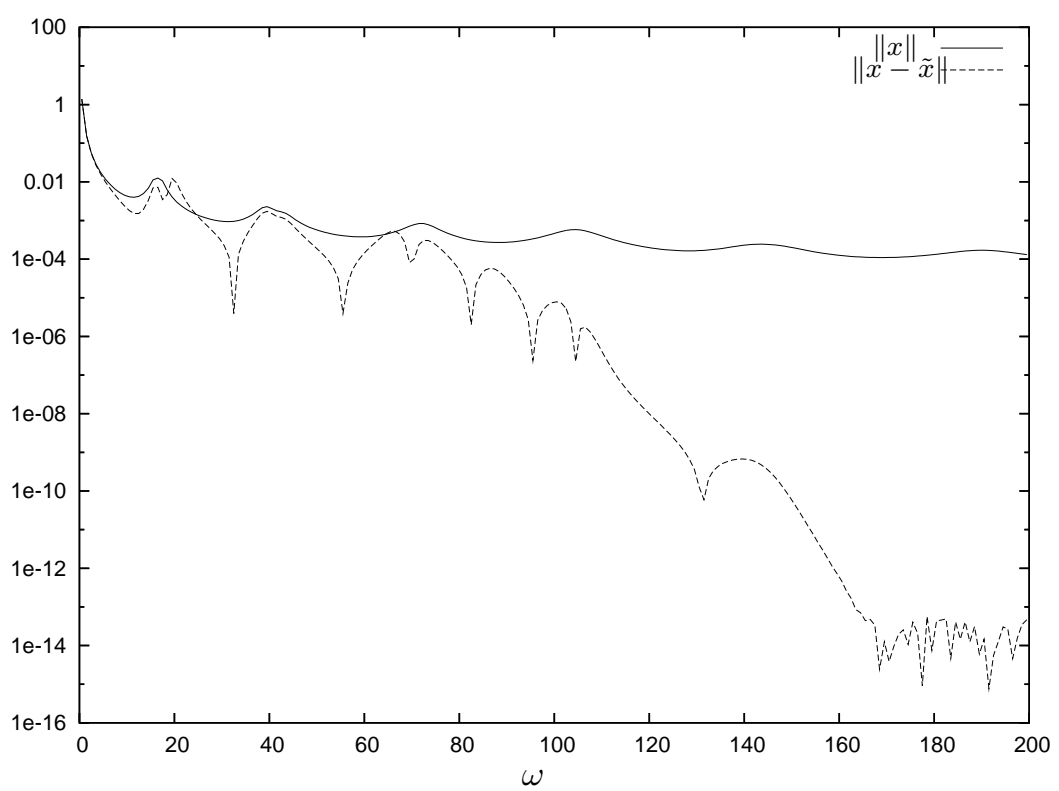

Figure 3: Computational results for the Lanczos method for the windscreen problem with $k=20$

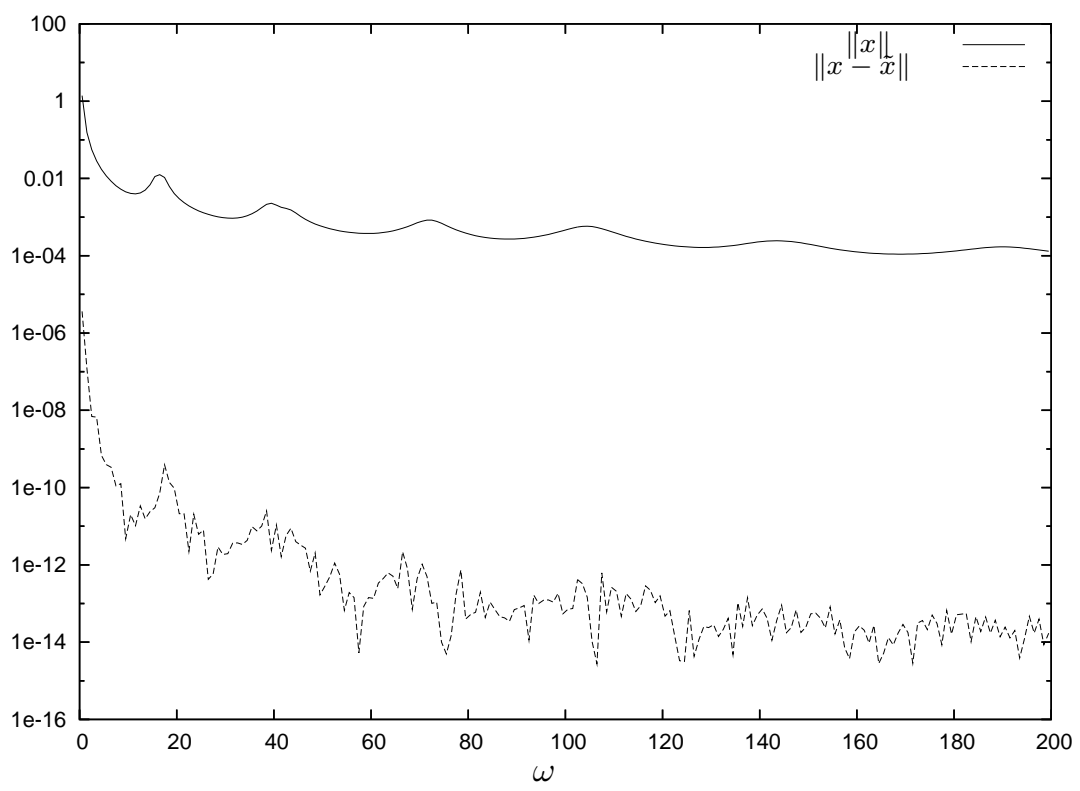

Figure 4: Computational results for the Lanczos method for the windscreen problem with $k=20$ 
[2] J. Angeles and S. Ostrovskaya. The proportional-damping matrix of arbitrarily damped linear mechanical systems. Journal of Applied Mechanics, 69:649-656, 2002.

[3] Z. Bai and R. Freund. A partial Padé-via-Lanczos method for reduced-order modeling. Linear Alg. Appl., 332-334:141-166, 2001.

[4] Z. Bai, K. Meerbergen, and Y. Su. Arnoldi methods for structure-preserving dimension reduction of secondorder dynamical systems, pages 173-189. Springer, Heidelberg, 2005.

[5] Z. Bai and Y. Su. Dimension reduction of second-order dynamical systems via a second-order arnoldi method. SIAM J. Matrix Anal. Applic., 26(5):1692-1709, 2005.

[6] K.-J. Bathe. Finite element procedure in engineering analysis. Prentice Hall, Englewood Cliffs, New Jersey, 1982.

[7] K.-J. Bathe and E.L. Wilson. Numerical Methods in Finite Element Analysis. Prentice-Hall, Englewood Cliffs, New Jersey, 1976.

[8] P. Benner, V. Mehrmann, and D.C. Sorensen. Dimension Reduction of Large-Scale Systems. Springer-Verlag, Berlin, Heidelberg, 2005.

[9] T. Ericsson. A generalised eigenvalue problem and the Lanczos algorithm. In J. Cullum and R.A. Willoughby, editors, Large Scale Eigenvalue Problems, pages 95-119. Elsevier Science Publishers BV, 1986.

[10] P. Feldman and R. W. Freund. Efficient linear circuit analysis by Padé approximation via the Lanczos process. IEEE Trans. Computer-Aided Design, CAD-14:639-649, 1995.

[11] R.W Freund. Krylov subspaces associated with higher-order linear dynamical systems. BIT, 45:495-516, 2005.

[12] A. Frommer and U. Glässner. Restarted GMRES for shifted linear systems. SIAM J. Sci. Comput., 19:15-26, 1998.

[13] T.K. Gaughey. Classical normal modes in damped linear dynamic systems. J. of Applied Mechanics, 27:269$271,1960$.

[14] T.K. Gaughey and M.E. O'Kelly. Classical normal modes in damped linear systems. J. of Applied Mechanics, Transaction of the ASME, 32:583-588, 1965.

[15] R.G. Grimes, J.G. Lewis, and H.D. Simon. A shifted block Lanczos algorithm for solving sparse symmetric genera lized eigenproblems. SIAM J. Matrix Anal. Applic., 15:228-272, 1994.

[16] L. Komzsik. The Lanczos method. SIAM, Philadelphia, 2003.

[17] Q. Luo and L. Tong. A sequential linear least squares algorithm for tracking dynamic shapes of smart structures. Intern. J. Numerical Methods in Engineering, 67:66-88, 2006.

[18] M. Malhotra and P. M. Pinsky. Efficient computation of multi-frequency far-field solutions of the Helmholtz equation using Padé approximations. J. Comput. Acoustics, 8(1):223-240, 2000.

[19] O. Marques. BLZPACK : Description and users's guide. Technical Report TR/PA/95/30, CERFACS, Toulouse, France, 1995.

[20] K. Meerbergen. The solution of parametrized symmetric linear systems. SIAM J. Matrix Anal. Appl., 24(4):1038-1059, 2003.

[21] V. Simoncini and E. Gallopoulos. Transfer functions and resolvent norm approximation of large matrices. ETNA, 7:190-201, 1998. 